\title{
Accuracy of SD Malaria Ag P.f/Pan® As A Rapid Diagnosis Test of Malaria in French Amazonia
}

jean marc pujo ( $\square$ tamac1966@gmail.com )

Centre Hospitalier de l'Universite de Montreal Centre de Recherche https://orcid.org/0000-0001-8146-3362

\section{Sarah Lemmonier}

Centre Hospitalier Andrée Rosemon: Centre Hospitalier Andree Rosemon

\section{Stéphanie Houcke}

Centre Hospitalier Andrée Rosemon: Centre Hospitalier Andree Rosemon

\section{Alexis Frémery}

Centre Hospitalier Andrée Rosemon: Centre Hospitalier Andree Rosemon

\section{Denis Blanchet}

Centre Hospitalier de Cayenne: Centre Hospitalier Andree Rosemon

\section{Felix Djossou}

Centre Hospitalier Andrée Rosemon: Centre Hospitalier Andree Rosemon

\section{Hatem Kallel}

Centre Hospitalier Andrée Rosemon: Centre Hospitalier Andree Rosemon

\section{Magalie Demar}

Centre Hospitalier Andrée Rosemon: Centre Hospitalier Andree Rosemon

\section{Research}

Keywords: malaria, rapid diagnosis tests accuracy, PfHRP2 gene deletion

Posted Date: May 4th, 2021

DOI: https://doi.org/10.21203/rs.3.rs-449138/v1

License: (c) (1) This work is licensed under a Creative Commons Attribution 4.0 International License. Read Full License 


\section{Abstract}

\section{Background}

French Guiana (FG) is, with Mayotte island, one of the two French overseas territories where malaria is still endemic. Ten years ago, French health authorities implemented a strategy to eradicate malaria disease in the region. The current incidence rate is $0,74 \%$ inhabitants, and Plasmodium Vivax is widely predominating even though Plasmodium Falciparum is still present due to imported cases, mainly from the Africa continent. In FG, RDT (SD Malaria Ag P.f/Pan ${ }^{\circledR}$ ) test is based on detection of pan-pLDH, PfHRP2, and PfHRP3 antigens. While, in South America, the share of deletion of PfHRP2 gene is significantly increasing. So, we wondered if RDTs remained reliable in this context.

\section{Methods}

Our study is a retrospective analysis conducted over four years and analyzed 12880 rapid diagnosis tests (RDTs) whose result was compared with Blood Film Tests (BFTs) sampled for malaria diagnosis.

\section{Results}

The global assessment of the diagnosis accuracy of RDT in the diagnosis of malaria shows PPV (Positive Predictive Value) and NPV (Negative Predictive Value) more than 95\%, except for PPV of RDT in the diagnosis of malaria to $P$ falciparum (88\%). Overall, the concordance rate between RDT and BFT (positive/positive; negative/negative) was 99.5\%. The PPV of the RDT in the follow-up of patients diagnosed with $P$ falciparum was the lowest during the 28 first days after diagnosis. The PPV of the RDT in the follow-up of patients diagnosed with $P$ vivax was the lowest during the 21 first days after diagnosis. The global sensitivity of SD Malaria Ag P.f/Pan® test was, on average, $96 \%$ (88.2 - 1) for $P$ falciparum and $93 \%(90.6-94.2)$ for $P$ vivax. The global specificity was high, with a rate of $99.8 \%(99.5-1)$ for all species included.

\section{Conclusion}

SD Malaria Ag P.f/Pan ${ }^{\circledR}$ is still a reliable rapid test used for the first-line diagnosis in remote healthcare centers. The test reading should be nuanced by the recent medical history of patients and the date of arrival in FG. RDT result has always to be confirmed by BFT that is still the gold standard for the malaria diagnosis. FG is still a PfHRP2 gene deletion-free area.

\section{Background}

WHO ranked malaria as a communicable disease among world public health priorities. In 2017 , malaria was still the most prevalent parasitic disease, with 1,4 billion people remaining at risk worldwide [2]. The year after, the number of malaria cases was estimated at 228 million, with 405.000 deaths worldwide, with a large predominance on the African continent. The global incidence declined between 2010 and 2018 with 57 cases/1000 inhabitants against 71 cases/1000 in 2010 then stayed at the same level until 2018. In the Americas region, the Malaria incidence is increasing since 2016, mainly due to the epidemic situation in Venezuela [3,4]. Plasmodium Vivax is predominating in the Americas Region, accounting for $75 \%$ of malaria cases [3]. With Mayotte island, French Guiana (FG) is one of the two French overseas territories where malaria is still endemic [5]. In FG, efforts to fight malaria since the 1950s and the control strategies implemented to the Guiana Shield [6] have led to a significant reduction of yearly recorded cases [7]. In 2019, the number of recorded malaria cases was the lowest for more than ten years, with 212 identified patients in the public IT healthcare system. Thus, the malaria incidence rate is $0,74 \%$ o inhabitants [8]. Previously in FG, Plasmodium falciparum was accountable for the majority of cases. However, within the last twenty years, the 
distribution of Plasmodium species changed with a vast rate of diagnosed Plasmodium Vivax [7,9]. Despite a very encouraging figure with very low malaria transmission in the coastal and urban areas, the malaria risk is still endemic in FG. The country's inland sites experience higher levels mainly related to socially marginalized and isolated populations.

Among lab methods used for malaria parasite detection and exposure, only rapid diagnosis tests (RDT) and blood films test (BFT) are currently available for daily practice. Molecular methods are recommended for the submicroscopic detection of malaria parasites. Serology is recommended for the survey and elimination assessment when the parasite prevalence reaches a level lower than 1\%. RDT is based on detection of pan-pLDH, PfHRP2 and PfHRP3 antigens. However, there is an increasing rate of deletion of the PfHRP2 gene worldwide, threatening the ability to diagnose patients infected with Plasmodium falciparum, responsible for numerous false negatives in RDT results (https://www.who.int/malaria/publications/). In South America, the distribution of PfHRP2 gene deletion varies with free countries and areas where the deletion rate is over 30\%. In French Guiana, the unique study performed on 140 isolates of Plasmodium falciparum did not find PfHRP2 deletion but 4.5\% PfHRP3 deletion [10].

With a unique study including a small cohort of RDTs and the knowledge from surrounding countries, we wondered if results were still reliable. We gathered the large number of RDTs from 2016 to 2019 to get a significant level for the validation of statistics. This study aims to evaluate the diagnostic relevance of RDT used in the diagnosis of malaria in French Guiana.

\section{Methods}

Our study is a retrospective analysis conducted over four years (January 2016 to December 2019) in the microbiology laboratory of the Cayenne General Hospital. It includes all RDT and BFT sampled for malaria diagnosis. Cayenne General Hospital is a 742-beds health facility that provides first-line medical care for an urban population of 150,000 inhabitants. It manages 18 delocalized prevention and healthcare centers providing care for almost 50,000 inhabitants. Thereby, it is also a referral center for a larger population coming from all over French Guiana and the border countries.

\section{Data collection}

Data were collected from the computerized database of the microbiology laboratory of the Cayenne General Hospital. They include the date of the tests, the result of the RDT, and concomitant BFT.

In a first step, we included all files with a RDT and a concomitant BFT. We excluded all files with a BFT without a concomitant RDT, and we put apart all files where concomitant RDT/BFT were sampled during the six months following the diagnosis of malaria.

In a second step, we analyzed all files where concomitant RDT/BFT were sampled during the six months following malaria diagnosis.

\section{Microbiological technique}

RDT was based on the SD Malaria Ag P.f/Pan® (Standard Diagnostics Inc.), which detects the presence of pan-pLDH and PfHRP2 antigens [10].

BFT is the gold standard for the diagnosis of malaria. It was based on microscopic examination of blood. It is the mainstay of malaria diagnosis [11]. Two sorts of blood film are traditionally used. Thin films allow species identification with better identification of the parasite. Thick films allow the screening of a larger blood volume and are 
about eleven times more sensitive than thin films. Both smears should be used when attempting to make a definitive diagnosis of malaria [15]. Indeed, thick films allow the diagnosis of infection with a low level of parasites, whereas thin films allow better identification of the responsible parasite.

Thick and thin blood films were prepared within one hour of blood collection. Thick blood films were stained with Giemsa diluted at $10 \%$, while thin blood films were stained using a rapid method (RALH 555, RAL Diagnostics). Two hundred fields of the thin blood film were examined before classifying the thin smear-negative, then 1000 counted white blood cells (WBCs) from the thick smear were observed before classifying the sample as negative [10]. The parasite density estimation was based on an assumed $6,000 \mathrm{WBC} / \mathrm{ml}$ of blood [10].

\section{Statistical analysis}

Results are reported as mean and standard deviation or numbers with percentages. We calculated the sensitivity, specificity, positive and negative predictive values, Youden test, and the Q coefficient of Yule to assess the diagnosis value of RDT in the diagnosis of confirmed malaria by BFT. All statistical analyses were carried out with Excel (2010 Microsoft corporation, Redmond, USA) and IBM SPSS Statistics for Windows, version 24 (IBM Corp., Armonk, NY, USA).

\section{Ethical consideration}

Our study is a retrospective that did not require individual consent according to the French law regarding research conforming to MR-003 (JORF no. 0160 du 13 juillet 2018. texte no. 109). Our database has been registered at the Commission National de l'Informatique et des Libertés (registration $n^{\circ} 2219819$ ), in compliance with French law on electronic data sources

\section{Results}

During the study period, 12984 samples of blood films for the diagnosis of malaria were ordered. A concomitant BFT and RDT were sampled in 12880 cases. About them, 10873 cases (84.4\%) fulfilled our inclusion criteria (Figure 1).

The average number of RDTs and BFTs per year was $2718 \pm 394$ tests. The average number of RDTs and BFTs per month was $1073 \pm 105$ tests. RDT was performed in 10873 cases and was positive in 773 cases (7.1\%) with identification of $P$ falciparum in 125 cases (16.1\% of positive tests) and to $P$ vivax in 648 cases (83.9\% of positive tests). BFT was performed in 10873 cases and was positive in 791 cases (7.3\%) with identification of $P$ falciparum in 105 cases (13.3\% of positive tests), and to $P$ vivax in 673 cases ( $85.1 \%$ of positive tests), to both of them in 10 cases ( $1.3 \%$ of positive tests), and $P$ ovale in 2 cases ( $0.3 \%$ of positive tests).

The global assessment of the diagnosis accuracy of RDT in the diagnosis of malaria shows PPV and NPV more than $95 \%$, except for PPV of RDT in the diagnosis of malaria to $P$ falciparum (88\%). Figure 2 shows the diagnosis accuracy of RDT in the diagnosis of malaria independently of the plasmodium species identification.

The yearly assessment of the diagnosis accuracy of RDT in the diagnosis of malaria, of malaria to P falciparum, and of malaria to $P$ vivax is reported in Table 1. The yearly assessment of the diagnosis accuracy of RDT in the diagnosis of malaria to $P$ falciparum shows a drop-down in the PPV in 2017 but an increase after that (Figure 3).

Table 1: Evolution of RDT quality criteria yearly 


\begin{tabular}{|c|c|c|c|c|c|c|c|c|c|c|c|c|}
\hline Year & $\begin{array}{l}\text { Comparaison } \\
\text { variable }\end{array}$ & $\mathrm{Nb}$ & $\mathrm{TP}$ & FP & TN & FN & Ss & Sp & PPV & NPV & Q & Youden \\
\hline \multirow[t]{3}{*}{2016} & RDT & 3276 & 104 & 6 & 3164 & 2 & 0.981 & 0.998 & 0.945 & 0.999 & 1.000 & 0.979 \\
\hline & \multicolumn{2}{|c|}{ RDT /P falciparum } & 30 & 5 & 3237 & 4 & 0.882 & 0.998 & 0.857 & 0.999 & 1.000 & 0.881 \\
\hline & \multicolumn{2}{|l|}{ RDT / P vivax } & 71 & 4 & 3196 & 5 & 0.934 & 0.999 & 0.947 & 0.998 & 1.000 & 0.933 \\
\hline \multirow[t]{3}{*}{2017} & $\mathrm{RDT}$ & 2462 & 297 & 10 & 2144 & 11 & 0.964 & 0.995 & 0.967 & 0.995 & 1.000 & 0.960 \\
\hline & \multicolumn{2}{|c|}{ RDT /P falciparum } & 37 & 9 & 2415 & 1 & 0.974 & 0.996 & 0.804 & 1.000 & 1.000 & 0.970 \\
\hline & \multicolumn{2}{|l|}{ RDT /P vivax } & 258 & 3 & 2185 & 16 & 0.942 & 0.999 & 0.989 & 0.993 & 1.000 & 0.940 \\
\hline \multirow[t]{3}{*}{2018} & $\mathrm{RDT}$ & 2714 & 265 & 3 & 2430 & 16 & 0.943 & 0.999 & 0.989 & 0.993 & 1.000 & 0.942 \\
\hline & \multicolumn{2}{|c|}{ RDT /P falciparum } & 33 & 1 & 2680 & 0 & 1.000 & 1.000 & 0.971 & 1.000 & 1.000 & 1.000 \\
\hline & \multicolumn{2}{|l|}{ RDT /P vivax } & 232 & 2 & 2464 & 16 & 0.935 & 0.999 & 0.991 & 0.994 & 1.000 & 0.935 \\
\hline \multirow[t]{3}{*}{2019} & RDT & 2421 & 87 & 1 & 2324 & 9 & 0.906 & 1.000 & 0.989 & 0.996 & 1.000 & 0.906 \\
\hline & \multicolumn{2}{|c|}{ RDT /P falciparum } & 10 & 0 & 2411 & 0 & 1.000 & 1.000 & 1.000 & 1.000 & 1.000 & 1.000 \\
\hline & \multicolumn{2}{|l|}{$\mathrm{RDT} / \mathrm{P}$ vivax } & 77 & 1 & 2335 & 8 & 0.906 & 1.000 & 0.987 & 0.997 & 1.000 & 0.905 \\
\hline
\end{tabular}

RDT: Rapid diagnosis tests, Nb: Number of cases, TP: True positive, FP: False positive, TN: True negative, FN: False negative, Ss: Sensitivity, Sp: Specificity, PPV: Positive Predictive Value, NPV: Negative Predictive Value

Overall, the concordance rate between RDT and BFT (positive/positive; negative/negative) was $99.5 \%$. It was the highest in 2016 (99.8\%) and the lowest in 2017 (99.1\%) (Figure 4).

The concordance rate between RDT and BFT (positive/positive; negative/negative) in the diagnosis of $P$ falciparum was $99.8 \%$. It was at $99.6 \%$ in 2017 (the lowest) and 100\% in 2018-2019. The concordance rate between RDT and BFT (positive/positive; negative/negative) in the diagnosis of $P$ vivax was $99.3 \%$. It was at $99.1 \%$ in 2017 (the lowest) and $99.5 \%$ in 2016 and 2019.

During the study period, 2007 cases (15.6\%), the RDT and the BFT were performed as part of the follow-up of patients diagnosed with malaria. The PPV of the RDT was the lowest during the 42 first days (6 weeks) after diagnosis (Figure 5).

The PPV of the RDT in the follow-up of patients diagnosed with $P$ falciparum was the lowest during the 28 first days after diagnosis. The PPV of the RDT in the follow-up of patients diagnosed with $P$ vivax was the lowest during the 21 first days after diagnosis (Table 2).

Table2: Evolution of RDT quality criteria during four months all species included 


\begin{tabular}{|llllllllllll|}
\hline Time (days) & Nb & TP & FP & TN & FN & Ss & Sp & PPV & NPV & Q & Youden \\
\hline 0 to 7 & 992 & 116 & 61 & 803 & 12 & 0.906 & 0.929 & 0.655 & 0.985 & 0.984 & 0.836 \\
\hline 8 to 14 & 221 & 6 & 7 & 208 & 0 & 1.000 & 0.967 & 0.462 & 1.000 & 1.000 & 0.967 \\
\hline 15 to 21 & 123 & 9 & 4 & 109 & 1 & 0.900 & 0.965 & 0.692 & 0.991 & 0.992 & 0.865 \\
\hline 22 to 28 & 91 & 20 & 0 & 70 & 1 & 0.952 & 1.000 & 1.000 & 0.986 & 1.000 & 0.952 \\
\hline 28 to 35 & 76 & 25 & 1 & 48 & 2 & 0.926 & 0.980 & 0.962 & 0.960 & 0.997 & 0.906 \\
\hline 36 to 42 & 64 & 19 & 0 & 44 & 1 & 0.950 & 1.000 & 1.000 & 0.978 & 1.000 & 0.950 \\
\hline 43 to 49 & 46 & 10 & 1 & 35 & 0 & 1.000 & 0.972 & 0.909 & 1.000 & 1.000 & 0.972 \\
\hline 50 to 56 & 33 & 7 & 0 & 26 & 0 & 1.000 & 1.000 & 1.000 & 1.000 & 1.000 & 1.000 \\
\hline 57 to 90 & 151 & 29 & 0 & 119 & 3 & 0.906 & 1.000 & 1.000 & 0.975 & 1.000 & 0.906 \\
\hline 91 to 120 & 86 & 14 & 0 & 72 & 0 & 1.000 & 1.000 & 1.000 & 1.000 & 1.000 & 1.000 \\
\hline
\end{tabular}

RDT: Rapid diagnosis tests, Nb: Number of cases, TP: True positive, FP: False positive, TN: True negative, FN: False negative, Ss: Sensitivity, Sp: Specificity, PPV: Positive Predictive Value, NPV: Negative Predictive Value

\section{Discussion}

Even though FG is still not concerned by this FpHPR2 gene deletion [10], the phenomenon of immigration from neighboring countries and over might introduce species carrying the genetic mutation. Our study aimed to assess the accuracy of SD Malaria Ag P.f/Pan ${ }^{\circledR}$ test, which is the RDT currently used in remote healthcare centers of FG, in the rapid diagnosis of malaria. The SD Malaria Ag P.f/Pan® test was sufficiently accurate for diagnosing malaria in suspected patients and the routine monitoring and the detection of passive cases in malaria low transmission areas (Orientation note about malaria diagnosis in the context of low transmission rate, September 2014, WHO). Diagnosing malaria is an emergency to early introduce a curative treatment and prevent an adverse evolution that, in some cases, could lead to death. Microscopic blood smear examination is still the gold standard, but high-quality RDTs can be used, at first, due to their efficacy, large availability, and cheap cost (WHO. Quality assurance for microscopy: malaria microscopy quality assurance manual. Genève, 2009). In FG remote areas, the only way to quickly diagnose malaria disease remains RDTs. The choice of the SD Malaria Ag P.f/Pan® in FG was based on the comparison of performance provided by the WHO reports but not on entomological studies made in FG.

(https://www.who.int/diagnostics_laboratory/evaluations/pq-list/malaria/public_report/en/).

In FG, the implementation of a malaria control strategy allowed to reduce the number of recorded malaria cases by 82\% during the last ten years, with 3344 cases in 2009 and 212 cases in 2019 (https://www.guyane.ars.sante.fr). In 2011, among 1209 cases reported $P$ falciparum and $P$ vivax represented respectively $31 \%$ and 68.5\% [10]. In 2019, among the 212 recorded malaria cases, only $6 \%$ were $P$ falciparum (13 cases). Forty-two cases were hospitalized, and only 2 cases developed a complicated clinical picture (one with $P$ falciparum and one with $P$ vivax)

(https://www.guyane.ars.sante.fr/paludisme-1). In our study, the average share of confirmed (BFT test) $P$ falciparum decreased significantly with $13.3 \%$ of positive tests (minus $17.7 \%$ ), while confirmed $P$ vivax cases raised with $85.1 \%$ of positive tests (plus 16.6\%) (https://www.guyane.ars.sante.fr/system/files/2017-06/palu\%20plan\%202015-2018.pdf).

Our study recorded all RDT's performed and compared them to BFT regardless of whether it was a first-line diagnostic test or a monitoring test during the curative treatment. The global sensitivity of SD Malaria Ag P.f/Pan® test was, on 
average, $96 \%(88.2-1)$ for $P$ falciparum and $93 \%(90.6-94.2)$ for $P$ vivax. The sensitivity can be affected by a low rate of parasitemia in the bloodstream [1]. The global specificity was high, with a rate of $99.8 \%(99.5-1)$ for all species included. In our database, among 12880 concomitant RDT and BFT tests, 10873 tests (84.4\%) represented first-line diagnosis tests, and 2007 RDTs were performed during the follow-up period. The latter affected results of the VVP, which was on average $97 \%(94.5$ - 98.9) but significantly different for $P$ falciparum and $P$ vivax with respectively $88 \%(80.4-1)$ and $98 \%(94.7-99.1)$.

Most of RDTs performed during the follow-up period were done during the two first years, which can explain the annual variation of the PPV. Furthermore, there is a significant variation of the global PPV week after week during the first 28 days of our study. It is also explained by the number of false positives related to the persistence of the proteinencoding for Plasmodium in the blood. In our study, we have not collected clinical information. Consequently, we were not able to determine if there were an immunological factor or infectious agents to explain the calculated PPV [12,13]. Thus, a positive result of the RDT must be confirmed by a BFT. On the ground, there is a real risk of not making the correct diagnosis by placing too much importance on the rapid test result in case of positivity.

The negative predictive value was, on average, $99.7 \%$ (99.3 - 99.5) without a significant difference between $P$ falciparum and $P$ vivax. A low density of parasitemia can affect the accuracy of the NPV [14]. It was not the case in our study where we did not find false-negative results. We can deduce that the negativity of SD Malaria Ag P.f/Pan $\AA$, used as a first-line diagnosis test, allowed to reasonably eliminate malaria diagnosis even though perform BFT remains the gold standard.

In our study, 2007 concomitant RDT/BFT tests were carried during the follow-up period of patients during treatment. Our results have shown that the RDT result was still in the majority positive up to 28 days, even though parasites were no longer detectable with BFT. The literature emphasizes a remaining RDT positivity up to 63 days after treatment. Previous findings mention that PfHRP2 RDTs are still positive after treatment for longer than combination or pLDH RDTs [15]. This is explained by a slower degradation of PfHRP2 compared to pLDH after parasite elimination [15,16]. Indeed, PfHRP2 antigen, which is progressively eliminated from the bloodstream, can be responsible for false positives $[17,18]$. In opposition, pLDH is quickly eliminated after one week from the bloodstream [19]. Thus, the initial parasite density influence false positives result and PfHRP2 persistence [16].

Furthermore, patients benefiting from ACT treatment have a higher probability of remaining positive to RDTs than those who received non-ACT drugs [16]. In practice, the persistence of positivity of RDT after treatment makes it obsolete for monitoring. Thus, the SD Malaria Ag P.f/Pan® test should not be used to assess the efficiency of the treatment set up in these conditions.

During the last two years, the majority of malaria cases in FG were autochthonous (83\% first-quarter 2019, 78\% firstquarter 2020) while the remaining part was from Brazil (range 9 to $14 \%$ ), Suriname (range 2 to $5 \%$ ), or Africa (range 2 to 7\%) [8]. Illegal gold miners (garimpeiros) inside the rainforest and native populations at border areas between Suriname and Brazil are at risk of malaria resurgence $[7,20,21]$. The epidemic of malaria experienced a pic in 2017 at the Amazonian border between FG and Brazil. Multifactorial causes were pointed out, such as migration matters from Brazil and Venezuela, local politics, logistics issues, and others [22]. Most cases occurred in forest areas except Saint Georges d'Oyapock at Brazil's border (Amapà region). https://www.guyane.ars.sante.fr/paludisme-1. In FG, the malaria incidence is low, so that all patients with positive RDT tests are considered as recently infected. However, in 2015, Orpal-1, a study carried out on a population of 421 gold miners, has shown that the wide majority were Brazilian citizens (93.8\%). This study has shown that the prevalence of carriage, determined by PCR, was $22.3 \%$ (95\% $\mathrm{Cl}$ : $18.3-$ 26.3) to $84 \%$ asymptomatic. Species identified were $P$ falciparum and $P$ vivax, $47.9 \%$ and $37.2 \%$, respectively with $10.6 \%$ co-infections. Thus, there is a real risk of periodic reintroduction of the disease in FG [23]. It is estimated that 
illegal gold miners are around 10.000 people (www. guyane.gouv.fr). Orpal-1 results with only $4.2 \%$ of the group, targeted as accountable for malaria cases spreading in FG, might be non-representative of the reality. Further investigations are required to clarify this key point.

By the way, in the Brazilian Amazon basin, the lack of PfHRP2 protein is very variable. The isolates collected in Acre state had the highest percentage of deletions with $31.6 \%$ (95\% Cl: 21.6-43.1), while in Rondonia state, the prevalence of deletion was only 3.3\% (95\% Cl: 0.4-11.5) and absent in Para state [24] but 100\% of deletion for Pf HRP3 [25]. In 2013, within the annual meeting of AMI/RAVDERA, in Lima, Peru, CDC reported in the Macapa region 9\% of PfHRP2 deletion (only 13 samples were screened) and $36 \%$ of PfHRP3 deletion. More globally, during the same meeting, CDC mentioned PfHPR2 deletion in 14\% of cases in Brazil, $14.1 \%$ in Suriname, 33.3\% in Peru, $7.5 \%$ in Colombia, and $4 \%$ in Bolivia [26]. Moreover, there is no accurate data about imported malaria cases except the distribution shares that may depend on immigration waves (www.guyane.ars.sante.fr). Besides, we do not know the immigration journey of patients and if they are coming from endemic countries with a significant rate of PfHPR2 deletion. In theory, it represents a risk of false-negative tests that should be better estimated by further studies [27].

\section{Conclusion}

SD Malaria Ag P.f/Pan ${ }^{\circledR}$ is still a reliable rapid test used for the first-line diagnosis in remote healthcare centers. However, it must no longer be used during the follow-up period of patients diagnosed with malaria. The test reading should be nuanced by the recent medical history of patients and the arrival date in FG. RDT result has always to be confirmed by BFT that is still the gold standard for the malaria diagnosis. FG is still a PfHRP2 gene deletion-free area. However, uncontrolled migratory flows might increase the risk of malaria reintroduction in the territory and thwart the strategy put in place with the possibility to import new species that might impact the efficiency of the test.

\section{Abbreviations}

ACT: Artemisinin-based combination therapy

BFT: Blood Film Tests

CDC: Centers for Disease Control and Prevention

FG: French Guiana

FN: False negative

FP: False positive

IT: Information Technology

$\mathrm{Nb}$ : Number

NPV: Negative Predictive Value

PPV: Positive Predictive Value

RDT: Rapid diagnosis tests

Sp: Specificity 
Ss: Sensitivity

TN: True negative

TP: True positive

WBC: White blood cells

WHO: World Health Organization

\section{Declarations}

- Ethics approval and consent to participate: Since our study is retrospective, it did not require individual consent according to the French law regarding research conforming to MR-003 (JORF no. 0160 du 13 juillet 2018. texte no. 109). Our database has been registered at the Commission National de I'Informatique et des Libertés (registration $\mathrm{n}^{\circ}$ 2219819), in compliance with French law on electronic data sources.

- Consent for publication: At admission to our hospital, an information booklet was distributed to all patients or their relatives stating that their data can be used for research and publication purposes and that they can object to that. No person was unconsented.

- Availability of data and materials: The datasets used and/or analyzed during the current study are available from the corresponding author on reasonable request.

- Competing interests: The authors declare that they have no competing interests.

- Funding: Not applicable

- Authors' contributions

JMP: Conceptualization, methodology, project administration, data curation, formal analysis.

SL: Data curation.

SH: Project administration.

AF: Data curation.

DB: Data curation, formal analysis.

FD: Conceptualization.

HK: Conceptualization, methodology, project administration, data curation, formal analysis.

MD: Conceptualization, methodology, project administration.

- Acknowledgements: Not applicable

- Authors' information (optional)

\section{References}


1. WHO. Malaria rapid diagnostic test performance. Results of WHO product testing of malaria RDTs: round 7 (20152016) [Internet]. WHO. World Health Organization; [cited 2020 Sep 13]. Available from:

http://www.who.int/malaria/publications/atoz/978924151268/en/

2. WHO. World malaria report 2018 [Internet]. WHO. World Health Organization; [cited 2020 Sep 13]. Available from: http://www.who.int/malaria/publications/world-malaria-report-2018/report/en/

3. World malaria report 2019 [Internet]. [cited 2020 Sep 13]. Available from: https://www.who.int/publications-detailredirect/9789241565721

4. Plan Maestro para el fortalecimiento de la respuesta al $\mathrm{VIH}$, la tuberculosis y la malaria en la República Bolivariana de Venezuela desde una perspectiva de salud pública - Venezuela (Bolivarian Republic of) [Internet]. ReliefWeb. [cited 2020 Sep 13]. Available from: https://reliefweb.int/report/venezuela-bolivarian-republic/planmaestro-para-el-fortalecimiento-de-la-respuesta-al-vih-la

5. Tarantola A, Eltges F, Ardillon V, Lernout T, Sissoko D, Kendjo E, et al. Malaria in France: Mainland and territories. Med Mal Infect. 2011;41:301-6.

6. Breeveld FJV, Vreden SGS, Grobusch MP. History of malaria research and its contribution to the malaria control success in Suriname: a review. Malar J. 2012;11:95.

7. Musset L, Pelleau S, Girod R, Ardillon V, Carvalho L, Dusfour I, et al. Malaria on the Guiana Shield: a review of the situation in French Guiana. Mem Inst Oswaldo Cruz. 2014;109:525-33.

8. ARS Guyane. Surveillance du paludisme 1er trimestre 2020: du 30 décembre 2019 au 29 mars 2020. Le Point Epidemio cellule Guyane. 2020;

9. Carme B, Ardillon V, Girod R, Grenier C, Joubert M, Djossou F, et al. Update on the epidemiology of malaria in French Guiana. Med Trop (Mars). 2009;69:19-25.

10. Trouvay M, Palazon G, Berger F, Volney B, Blanchet D, Faway E, et al. High performance of histidine-rich protein 2 based rapid diagnostic tests in French Guiana are explained by the absence of pfhrp2 gene deletion in P. falciparum. PLoS ONE. 2013;8:e74269.

11. Krafts KP, Hempelmann E, Oleksyn BJ. The color purple: from royalty to laboratory, with apologies to Malachowski. Biotech Histochem. 2011;86:7-35.

12. Lee J-H, Jang JW, Cho CH, Kim JY, Han ET, Yun SG, et al. False-positive results for rapid diagnostic tests for malaria in patients with rheumatoid factor. J Clin Microbiol. 2014;52:3784-7.

13. Mouatcho JC, Goldring JPD. Malaria rapid diagnostic tests: challenges and prospects. J Med Microbiol. 2013;62:1491-505.

14. Natama HM, Ouedraogo DF, Sorgho H, Rovira-Vallbona E, Serra-Casas E, Somé MA, et al. Diagnosing congenital malaria in a high-transmission setting: clinical relevance and usefulness of P. falciparum HRP2-based testing. Sci Rep. 2017;7:2080.

15. Dalrymple U, Arambepola R, Gething PW, Cameron E. How long do rapid diagnostic tests remain positive after antimalarial treatment? Malar J. 2018;17:228.

16. Houzé S, Boly MD, Le Bras J, Deloron P, Faucher J-F. Pf HRP2 and Pf LDH antigen detection for monitoring the efficacy of artemisinin-based combination therapy (ACT) in the treatment of uncomplicated falciparum malaria. Malar J. 2009;8:211.

17. Iqbal J, Siddique A, Jameel M, Hira PR. Persistent histidine-rich protein 2, parasite lactate dehydrogenase, and panmalarial antigen reactivity after clearance of Plasmodium falciparum monoinfection. J Clin Microbiol. 2004;42:4237-41. 
18. Biswas S, Tomar D, Rao DN. Investigation of the kinetics of histidine-rich protein 2 and of the antibody responses to this antigen, in a group of malaria patients from India. Ann Trop Med Parasitol. 2005;99:553-62.

19. Abba K, Kirkham AJ, Olliaro PL, Deeks JJ, Donegan S, Garner P, et al. Rapid diagnostic tests for diagnosing uncomplicated non-falciparum or Plasmodium vivax malaria in endemic countries. Cochrane Database Syst Rev. 2014;CD011431.

20. Douine M, Musset L, Corlin F, Pelleau S, Pasquier J, Mutricy L, et al. Prevalence of Plasmodium spp. in illegal gold miners in French Guiana in 2015: a hidden but critical malaria reservoir. Malar J. 2016;15:315.

21. Ardillon V, Carvalho L, Prince C, Abboud P, Djossou F. Bilans 2013 et 2014 de la situation du paludisme en Guyane. p 16-20. [Internet]. Bulletin de veille sanitaire Antilles-Guyane; 2015. Available from:

http://www.invs.sante.fr/fr/Publications-et-outils/Bulletin-de-veille-sanitaire/Tous-les-numeros/AntillesGuyane/Bulletin-de-veille-sanitaire-Antilles-Guyane.-n-1-Janvier-2015

22. Mosnier E, Roux E, Cropet C, Lazrek Y, Moriceau O, Gaillet M, et al. Prevalence of Plasmodium spp. in the Amazonian Border Context (French Guiana-Brazil): Associated Factors and Spatial Distribution. Am J Trop Med Hyg. 2020;102:130-41.

23. Cohen JM, Smith DL, Cotter C, Ward A, Yamey G, Sabot OJ, et al. Malaria resurgence: a systematic review and assessment of its causes. Malar J. 2012;11:122.

24. Viana GMR, Silva-Flannery L, Lima Barbosa DR, Lucchi N, do Valle SCN, Farias S, et al. Field evaluation of a real time loop-mediated isothermal amplification assay (RealAmp) for malaria diagnosis in Cruzeiro do Sul, Acre, Brazil. PLoS One. 2018;13:e0200492.

25. Rachid Viana GM, Akinyi Okoth S, Silva-Flannery L, Lima Barbosa DR, Macedo de Oliveira A, Goldman IF, et al. Histidine-rich protein 2 (pfhrp2) and pfhrp3 gene deletions in Plasmodium falciparum isolates from select sites in Brazil and Bolivia. PLoS ONE. 2017;12:e0171150.

26. Akinyi Okoth S, Abdallah JF, Ceron N, Adhin MR, Chandrabose J, Krishnalall K, et al. Variation in Plasmodium falciparum Histidine-Rich Protein 2 (Pfhrp2) and Plasmodium falciparum Histidine-Rich Protein 3 (Pfhrp3) Gene Deletions in Guyana and Suriname. PLoS ONE. 2015;10:e0126805.

27. Gendrot M, Fawaz R, Dormoi J, Madamet M, Pradines B. Genetic diversity and deletion of Plasmodium falciparum histidine-rich protein 2 and 3: a threat to diagnosis of P. falciparum malaria. Clin Microbiol Infect. 2019;25:580-5.

\section{Figures}




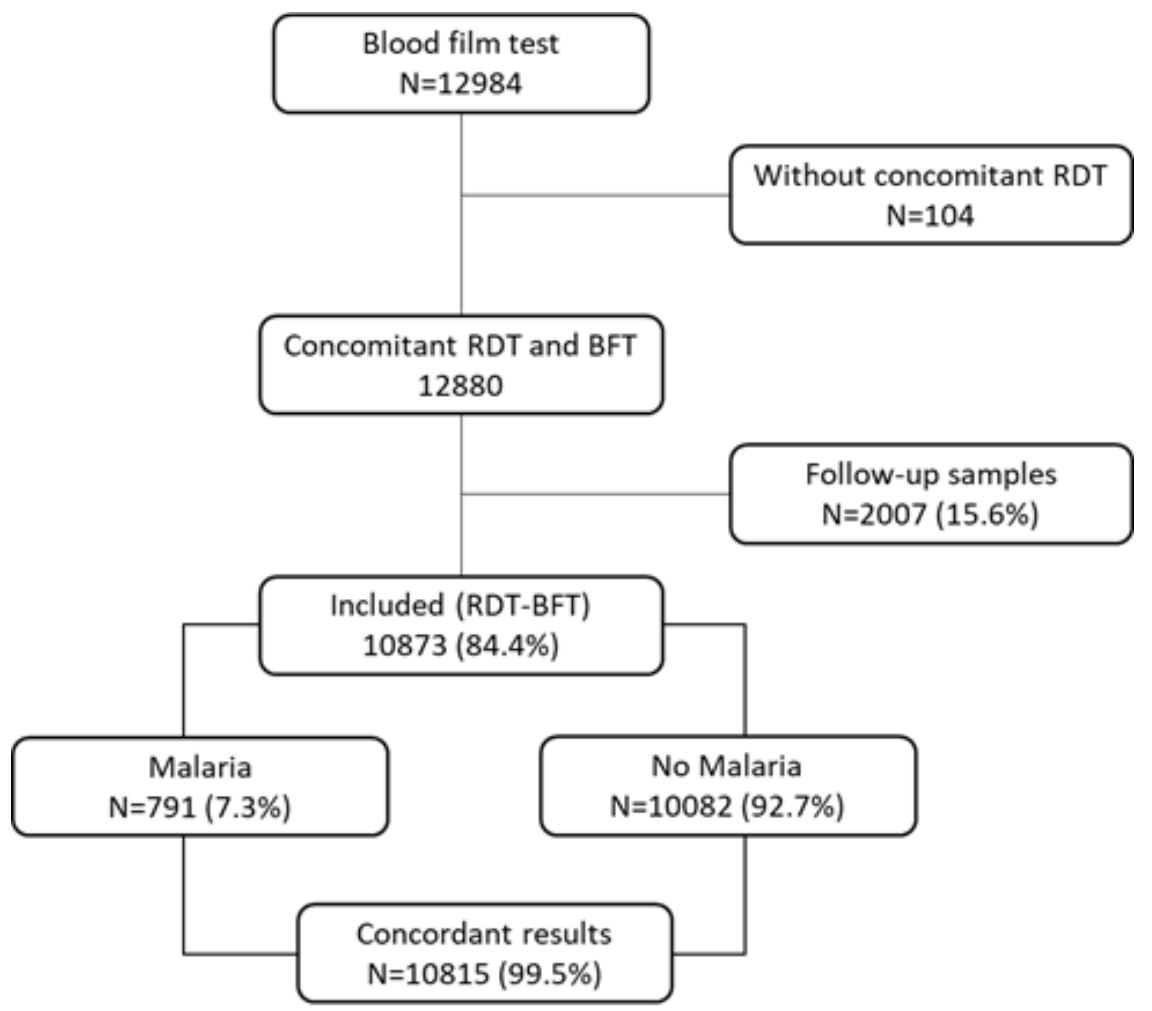

Figure 1

The flow chart of the study ( $\mathrm{N}=$ Number of cases)

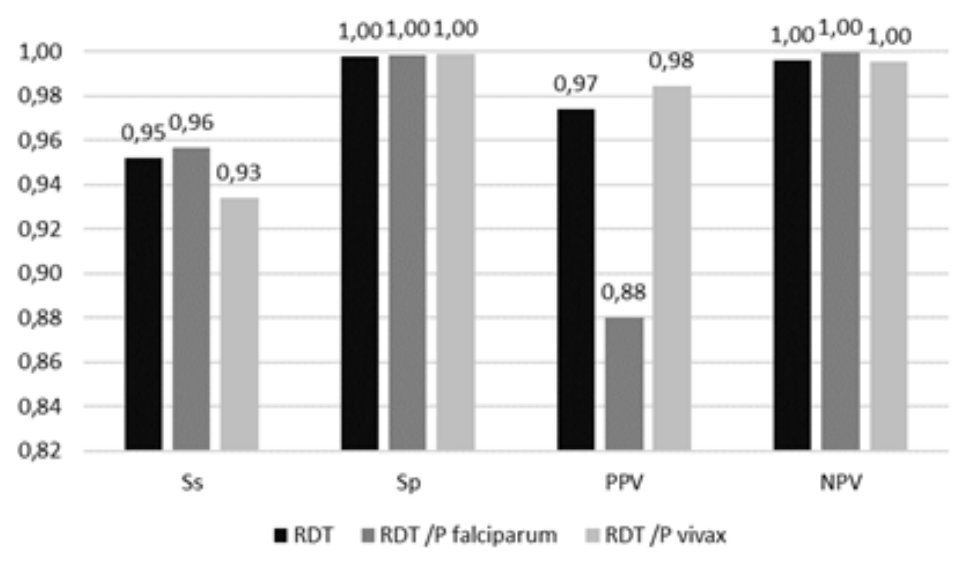

Figure 2

The diagnosis accuracy of RDT in diagnosing malaria according to the plasmodium species identification. (RDT: Rapid diagnosis tests, Ss: Sensitivity, Sp: Specificity, PPV: Positive Predictive Value, NPV: Negative Predictive Value) 


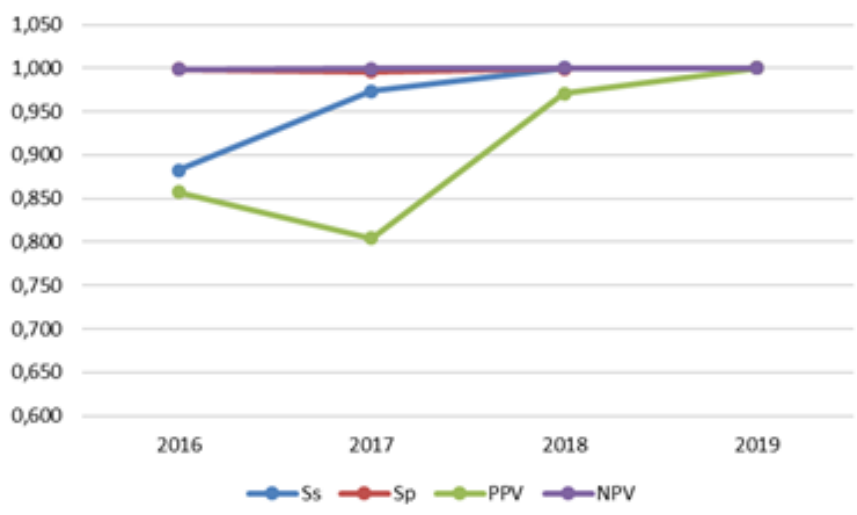

Figure 3

The diagnosis accuracy of RDT in the diagnosis of Plasmodium falciparum according to the year of the study. (RDT: Rapid diagnosis tests, Ss: Sensitivity, Sp: Specificity, PPV: Positive Predictive Value, NPV: Negative Predictive Value)

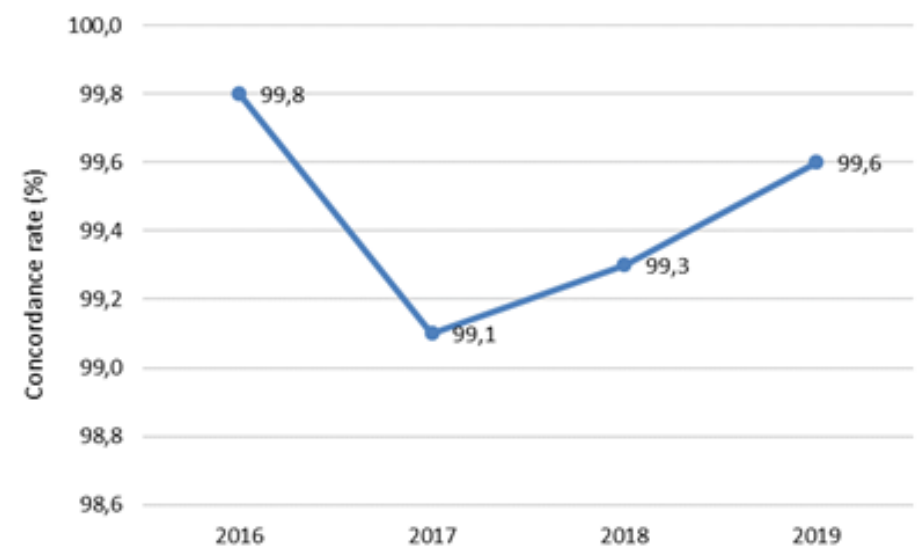

\section{Figure 4}

The concordance rate of RDT compared to BFT in the diagnosis of malaria independently of the plasmodium species identification according to the year of the study.

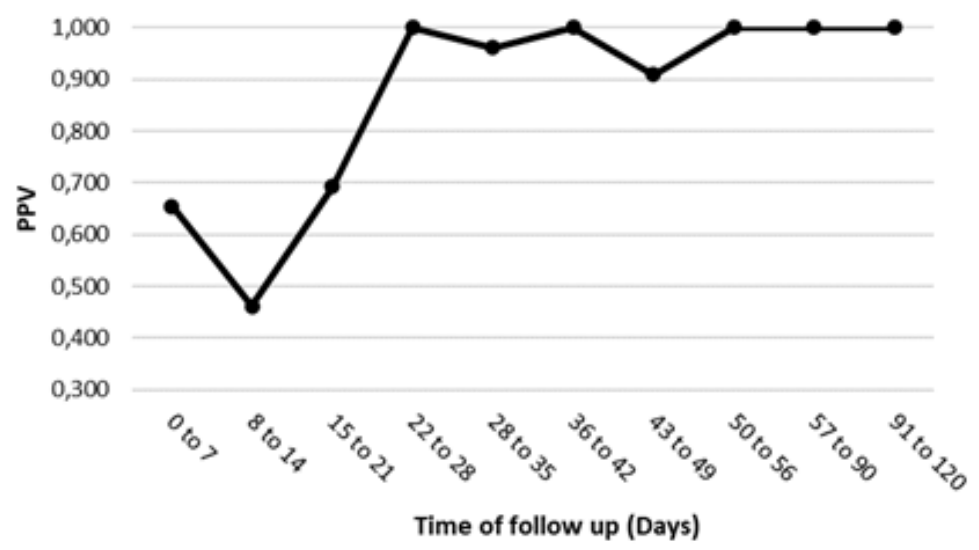

\section{Figure 5}

diagnosis accuracy of RDT in the follow-up period of malaria independently of the plasmodium species identification. 